Mahmoud A.E. Abdelrahman*

\title{
A note on Riccati-Bernoulli Sub-ODE method combined with complex transform method applied to fractional differential equations
}

https://doi.org/10.1515/nleng-2017-0145

Received October 23, 2017; revised December 12, 2017; accepted January 13, 2018.

\begin{abstract}
In this paper, the fractional derivatives in the sense of modified Riemann-Liouville and the RiccatiBernoulli Sub-ODE method are used to construct exact solutions for some nonlinear partial fractional differential equations via the nonlinear fractional Zoomeron equation and the $(3+1)$ dimensional space-time fractional mKDVZK equation. These nonlinear fractional equations can be turned into another nonlinear ordinary differential equation by complex transform method. This method is efficient and powerful in solving wide classes of nonlinear fractional order equations. The Riccati-Bernoulli SubODE method appears to be easier and more convenient by means of a symbolic computation system.
\end{abstract}

Keywords: Modified Riemann-Liouville derivative, RiccatiBernoulli Sub-ODE method, exact solution, fractional Zoomeron equation, $(3+1)$ dimensional space-time fractional $\mathrm{mKDV}$-ZK equation

MSC: 26A33, 34A08, 35A99, 35R11, 83C15, $65 \mathrm{Z} 05$

\section{Introduction}

Many physical phenomena such as mathematical biology, signal processing, optics, fluid mechanics, electromagnetic theory, etc., can be modeled using the fractional derivatives. Consequently, the investigation of exact solutions for FDEs turns out to be very useful in the study of scientific research. Moreover generalized forms of differential equations are described as fractional differential equations FDEs.

\footnotetext{
*Corresponding Author: Mahmoud A.E. Abdelrahman, Department of Mathematics, Faculty of Science, Mansoura University, 35516 Mansoura, Egypt, E-mail: mahmoud.abdelrahman@mans.edu.eg, mahmoud.abdelrahman1983@gmail.com, Tel +2 050 2242388; Fax +200502246781
}

Recent past, an strong attention has been purposed by the researchers concerning the fractional partial differential equations FDEs. For an interesting overview and more applications of nonlinear FDEs, we refer to [1-4].

However, even in most useful studies, there is no an efficient and general methods to solve them. Actually, various analytical and numerical methods to construct approximate and exact solutions of nonlinear FDEs have been put forward, such as the fractional sub-equation method [5, 6], the tanh-sech method [7], the $\left(\frac{G^{\prime}}{G}\right)$ - expansion method [8, 19], the first integral method [10], the modified Kudryashov method [11], the exponential function method [12,13] and others [14, 15].

The novelties of this paper are mainly exhibited in two aspects: First, we introduce a new method, which is not familiar, the so called Riccati-Bernoulli Sub-ODE method. We use this method to solve the nonlinear fractional Zoomeron equation and the $(3+1)$ dimensional space-time fractional mKDV-ZK equation. Moreover, we show that the proposed method gives infinite sequence of solutions. Second, we obtain new types of exact analytical solutions. Moreover comparing our results with other results, one can see that our results are new and most extensive.

Actually, the proposed two fractional equations have many applications in various fields of theoretical physics,applied mathematics and engineering such as control theory viscoelasticity, modelling heat transfer, control, diffusion, signal and image processing, and many other physical and engineering processes. In more details, the $(3+1)$ dimensional space-time fractional mKDV-ZK equation is derived for a plasma comprised of cool and hot electrons and a species of fluid ions, which have so many direct and indirect in engineering models. Furthermore the nonlinear fractional Zoomeron equation is a convenient model to display the novel phenomena associated with boomerons and trappons and further interesting engineering applications.

The Riccati-Bernoulli Sub-ODE technique has been used to solve some partial and fractional differential equations, see for example [16-21]. These works show that this 
method is efficacious, robust and adequate for solving further equations.

The rest of the paper is arranged as follows: In Section 2, we recall some basic definitions and notions dealing with fractional calculus theory, which are used in the sequel in this article. In Sections 4 and 3, two examples, namely the nonlinear fractional Zoomeron equation and the $(3+1)$ dimensional space-time fractional mKDVZK equation, are solved by the Riccati-Bernoulli Sub-ODE method. Conclusion will appear in Section 5.

\section{Preliminaries and notation}

We introduce some fundamental definitions and properties of fractional calculus theory, which turn to be very useful in order to complete this paper in a completely unified way. These are the Riemann-Liouville, the GrünwaldLetnikov, the Caputo and the modified Riemann-Caputo, Liouville derivative. The most commonly used definitions are the modified Riemann-Liouville and Caputo derivatives [22, 23]. Jumarie proposed a modified Riemann-Liouville derivative [24]. Firstly, we present some properties and definitions of the modified Riemann-Liouville derivative. Secondly, we give the description of the Riccati-Bernoulli Sub-ODE method.

Assume that $f(t)$ denotes a continuous $\mathbb{R} \rightarrow \mathbb{R}$ function (but not necessarily first-order differentiable). The Jumarie's modified Riemann-Liouville derivative is defined as

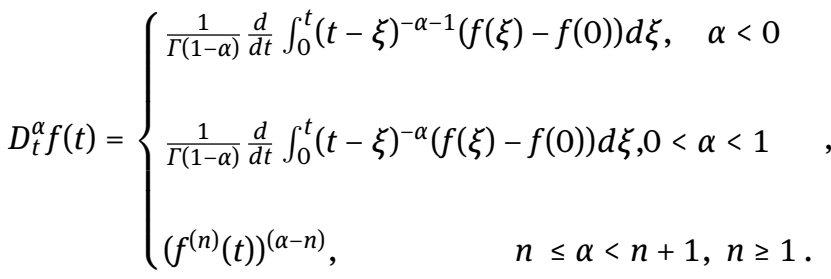

Important property of the fractional modified RiemannLiouville derivative is [25]

$$
D_{t}^{\alpha} t^{r}=\frac{\Gamma(1+r)}{\Gamma(1+r-\alpha)} t^{r-\alpha} .
$$

Step 1. Any nonlinear fractional differential equation in two independent variables $x$ and $t$ can be expressed in following form:

$$
G\left(u, D_{t}^{\alpha} u, D_{x}^{\alpha} u, D_{t}^{\alpha} D_{x}^{\alpha} u, D_{x}^{\alpha} D_{x}^{\alpha} u, \ldots\right)=0,
$$

where $0<\alpha \leq 1, D_{t}^{\alpha} u, D_{x}^{\alpha} u$ are modified RiemannLiouville derivative of $u$ and $G$ is a polynomial in $u(x, t)$ and its partial fractional derivatives.
Step 2. Using the traveling wave transformation

$$
u(x, t)=U(\xi), \quad \xi=\frac{k x^{\alpha}}{\Gamma(1+\alpha)}-\frac{\lambda t^{\alpha}}{\Gamma(1+\alpha)},
$$

where $k, \lambda$ are non zero constants and $1<\alpha \leq 1$. By using the chain rule,

$$
\begin{aligned}
& D_{t}^{\alpha} u=\sigma_{t}^{\prime} \frac{d U}{d \xi} D_{t}^{\alpha} \xi, \\
& D_{x}^{\alpha} u=\sigma_{x}^{\prime} \frac{d U}{d \xi} D_{x}^{\alpha} \xi,
\end{aligned}
$$

where $\sigma_{t}^{\prime}$ and $\sigma_{x}^{\prime}$ are called the sigma indexes, [26], without loss of generality, we can take $\sigma_{t}^{\prime}=\sigma_{x}^{\prime}=L$, where $L$ is a constant.

Superseding (2.4) with (2.2) and (2.5) into (2.3), the equation (2.3) transformed into the following ODE:

$$
H\left(U, U^{\prime}, U^{\prime \prime}, U^{\prime \prime \prime}, \ldots . .\right)=0,
$$

where prime denotes the derivation with respect to $\xi$.

Step 3. Based on the Riccati-Bernoulli Sub-ODE method [16-18], we assume that equation (2.6) has the following solution:

$$
U^{\prime}=a U^{2-n}+b U+c U^{n},
$$

where $a, b, c$ and $n$ are constants calculated later. From equation (2.7), we have

$$
\begin{gathered}
U^{\prime \prime}=a b(3-n) U^{2-n}+a^{2}(2-n) U^{3-2 n}+n c^{2} U^{2 n-1} \\
+b c(n+1) U^{n}+\left(2 a c+b^{2}\right) U, \\
U^{\prime \prime \prime}=\left(a b(3-n)(2-n) U^{1-n}+a^{2}(2-n)(3-2 n) U^{2-2 n}\right. \\
\left.+n(2 n-1) c^{2} U^{2 n-2}+b c n(n+1) U^{n-1}+\left(2 a c+b^{2}\right)\right) U^{\prime} .
\end{gathered}
$$

The exact solutions of equation (2.7), for an arbitrary constant $\mu$ are given as follow:

1. For $n=1$, the solution is

$$
U(\xi)=\mu e^{(a+b+c) \xi} .
$$

2. For $n \neq 1, b=0$ and $c=0$, the solution is

$$
U(\xi)=(a(n-1)(\xi+\mu))^{\frac{1}{n-1}} .
$$

3. For $n \neq 1, b \neq 0$ and $c=0$, the solution is

$$
U(\xi)=\left(\frac{-a}{b}+\mu e^{b(n-1) \xi}\right)^{\frac{1}{n-1}} .
$$


4. For $n \neq 1, a \neq 0$ and $b^{2}-4 a c<0$, the solution is

$$
\begin{aligned}
& U(\xi)= \\
& \left(\frac{-b}{2 a}+\frac{\sqrt{4 a c-b^{2}}}{2 a} \tan \left(\frac{(1-n) \sqrt{4 a c-b^{2}}}{2}(\xi+\mu)\right)\right)^{\frac{1}{1-n}}
\end{aligned}
$$

and

$$
\begin{aligned}
& U(\xi)= \\
& \left(\frac{-b}{2 a}-\frac{\sqrt{4 a c-b^{2}}}{2 a} \cot \left(\frac{(1-n) \sqrt{4 a c-b^{2}}}{2}(\xi+\mu)\right)\right)^{\frac{1}{1-n}} .
\end{aligned}
$$

5. For $n \neq 1, a \neq 0$ and $b^{2}-4 a c>0$, the solution is

$$
\begin{aligned}
& U(\xi)= \\
& \left(\frac{-b}{2 a}-\frac{\sqrt{b^{2}-4 a c}}{2 a} \operatorname{coth}\left(\frac{(1-n) \sqrt{b^{2}-4 a c}}{2}(\xi+\mu)\right)\right)^{\frac{1}{1-n}}
\end{aligned}
$$

and

$$
\begin{aligned}
& U(\xi)= \\
& \left(\frac{-b}{2 a}-\frac{\sqrt{b^{2}-4 a c}}{2 a} \tanh \left(\frac{(1-n) \sqrt{b^{2}-4 a c}}{2}(\xi+\mu)\right)\right)^{\frac{1}{1-n}} .
\end{aligned}
$$

6. For $n \neq 1, a \neq 0$ and $b^{2}-4 a c=0$, the solution is

$$
U(\xi)=\left(\frac{1}{a(n-1)(\xi+\mu)}-\frac{b}{2 a}\right)^{\frac{1}{1-n}} .
$$

\subsection{Bäcklund transformation}

When $U_{m-1}(\xi)$ and $U_{m}(\xi)\left(U_{m}(\xi)=U_{m}\left(U_{m-1}(\xi)\right)\right)$ are the solutions of equation (2.7), we have

$$
\begin{aligned}
\frac{d U_{m}(\xi)}{d \xi} & =\frac{d U_{m}(\xi)}{d U_{m-1}(\xi)} \frac{d U_{m-1}(\xi)}{d \xi} \\
& =\frac{d U_{m}(\xi)}{d U_{m-1}(\xi)}\left(a U_{m-1}^{2-n}+b U_{m-1}+c U_{m-1}^{n}\right),
\end{aligned}
$$

namely

$$
\frac{d U_{m}(\xi)}{a U_{m}^{2-n}+b U_{m}+c U_{m}^{n}}=\frac{d U_{m-1}(\xi)}{a U_{m-1}^{2-n}+b U_{m-1}+c U_{m-1}^{n}} .
$$

Integrating equation (2.18) once with respect to $\xi$, we get a Bäcklund transformation of equation (2.7) as follows:

$$
U_{m}(\xi)=\left(\frac{-c K_{1}+a K_{2}\left(U_{m-1}(\xi)\right)^{1-n}}{b K_{1}+a K_{2}+a K_{1}\left(U_{m-1}(\xi)\right)^{1-n}}\right)^{\frac{1}{1-n}},
$$

where $K_{1}$ and $K_{2}$ are arbitrary constants. We use equation (2.19) to obtain infinite sequence of solutions for equation (2.7), as well for equation (2.3).

\section{The nonlinear fractional Zoomeron equation}

We are concerned with the nonlinear fractional Zoomeron equation([27]),

$$
D_{t t}^{2 \alpha}\left[\frac{u_{x y}}{u}\right]-\left[\frac{u_{x y}}{u}\right]_{x x}+2 D_{t}^{\alpha}\left[u^{2}\right]_{x}=0, \quad 0<\alpha \leq 1,
$$

where $u(x, y, t)$ is the amplitude of the relevant wave mode.

Using the transformation

$$
\begin{gathered}
u(x, y, t)=U(\xi), \\
\xi=l x+\gamma y-\frac{w t^{\alpha}}{\Gamma(1+\alpha)},
\end{gathered}
$$

where $l, \gamma$ and $w$ are non zero constants and $0<\alpha \leq 1$.

Substituting (3.3) with (2.2) and (2.5) into (3.1), we have the ODE

$$
l \gamma w^{2}\left(\frac{U^{\prime \prime}}{U}\right)^{\prime \prime}-\gamma l^{3}\left(\frac{U^{\prime \prime}}{U}\right)^{\prime \prime}-2 l w\left(U^{2}\right)^{\prime \prime}=0 .
$$

Integrating this equation twice, with the second constant of integration is vanishing, we obtain

$$
l \gamma\left(w^{2}-l^{2}\right) U^{\prime \prime}-2 l w U^{3}-k U=0,
$$

where $k$ is a nonzero constant of integration.

Substituting equations (2.8) into equation (3.5), we obtain

$$
\begin{aligned}
& l \gamma\left(w^{2}-l^{2}\right)\left(a b(3-m) U^{2-m}+a^{2}(2-m) U^{3-2 m}+m c^{2} U^{2 m-1}\right. \\
& \left.+b c(m+1) U^{m}+\left(2 a c+b^{2}\right) U\right)-2 l w U^{3}-k U=0 . \\
& \text { Setting } m=0 \text {, equation (3.6) is reduced to } \\
& l \gamma\left(w^{2}-l^{2}\right)\left(3 a b U^{2}+2 a^{2} U^{3}+b c+\left(2 a c+b^{2}\right) U\right)-2 l w U^{3}-k U=0 .
\end{aligned}
$$

Equating each coefficient of $U^{i}(i=0,1,2,3)$ to zero, we have

$$
l \gamma\left(w^{2}-l^{2}\right) b c=0,
$$

$$
\begin{gathered}
l \gamma\left(w^{2}-l^{2}\right)\left(2 a c+b^{2}\right)-k=0, \\
3 l \gamma\left(w^{2}-l^{2}\right) a b=0, \\
2 l \gamma\left(w^{2}-l^{2}\right) a^{2}-2 l w=0 .
\end{gathered}
$$


Solving equations (3.8)-(3.11), we get

$$
b=0 \text {, }
$$

$$
\begin{aligned}
& a c=\frac{k}{2 l \gamma\left(w^{2}-l^{2}\right)}, \\
& a= \pm \sqrt{\frac{w}{\gamma\left(w^{2}-l^{2}\right)}},
\end{aligned}
$$

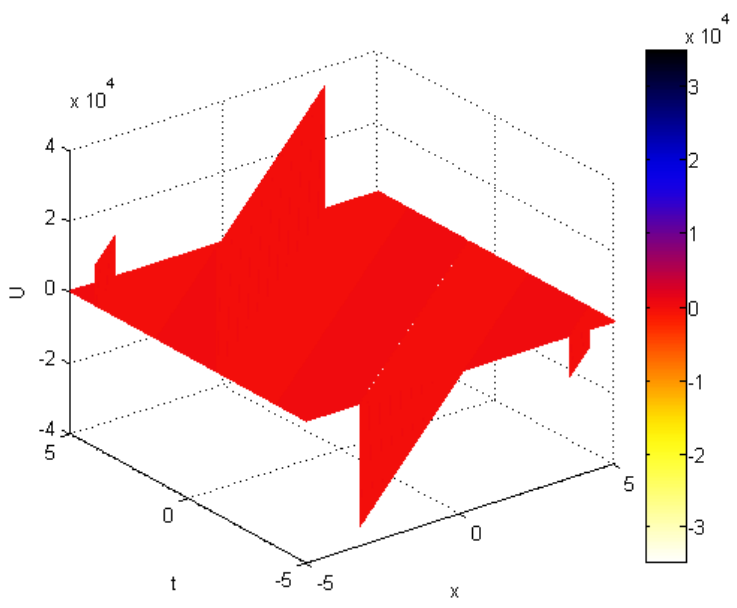

Fig. 1: The solution $U_{1}(x, 0, t)$ in (3.15) for $l=1.5, k=1, a=1, \mu=0, w=$ 2 and $-5 \leq t, x \leq 5$

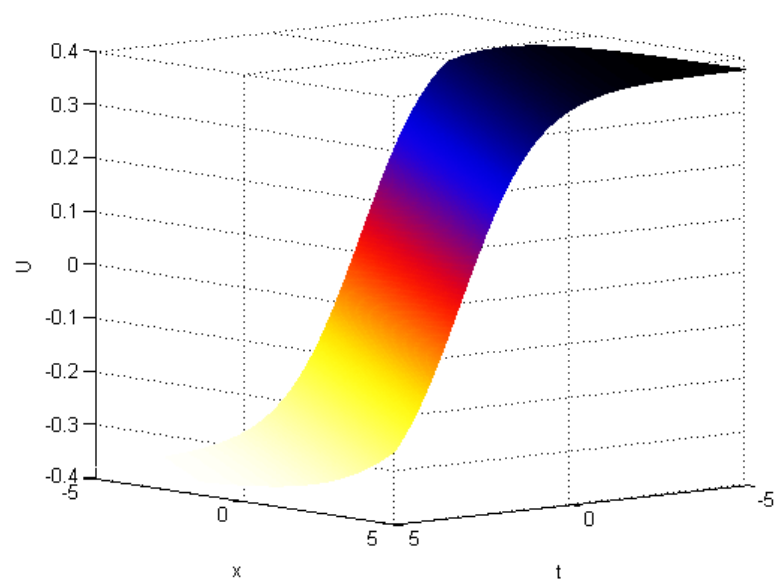

Fig. 2: The solution $U_{5}(x, 0, t)$ in (3.17) for $l=2, k=2, a=1, \mu=1, w=$ 3.5 and $-5 \leq t, x \leq 5$.

\section{Trigonometric function solutions:}

When $\frac{k}{l \gamma\left(w^{2}-l^{2}\right)}<0$, substituting equations (3.12)-(3.14) and (3.3) into equations (2.13) and (2.14), we get the exact solutions for equation (3.1),

$$
\begin{aligned}
& U_{1,2}(x, y, t)= \\
& \pm \sqrt{\frac{k}{2 w l}} \tan \left(\sqrt{\frac{k}{2 l w\left(w^{2}-l^{2}\right)}}\left(l x+\gamma y-\frac{w t^{\alpha}}{\Gamma(1+\alpha)}+\mu\right)\right)
\end{aligned}
$$

and

$$
\begin{aligned}
& U_{3,4}(x, y, t)= \\
& \pm \sqrt{\frac{k}{2 w l}} \cot \left(\sqrt{\frac{k}{2 l w\left(w^{2}-l^{2}\right)}}\left(l x+\gamma y-\frac{w t^{\alpha}}{\Gamma(1+\alpha)}+\mu\right)\right),
\end{aligned}
$$

where $l, k, \gamma, w, \mu$ are arbitrary constants and $0<\alpha \leq 1$. Figure 1 illustrated the solution $U_{2}$.

\section{Hyperbolic function solutions:}

When $\frac{k}{l \gamma\left(w^{2}-l^{2}\right)}>0$, substituting equations (3.12)-(3.14) and (3.3) into equations (2.15) and (2.16), we obtain exact solutions for equation (3.1),

$$
\begin{aligned}
& U_{5,6}(x, y, t)= \\
& \pm \sqrt{\frac{-k}{2 w l}} \tanh \left(\sqrt{\frac{k}{2 l w\left(l^{2}-w^{2}\right)}}\left(l x+\gamma y-\frac{w t^{\alpha}}{\Gamma(1+\alpha)}+\mu\right)\right)
\end{aligned}
$$

and

$$
\begin{aligned}
& U_{7,8}(x, y, t)= \\
& \pm \sqrt{\frac{-k}{2 w l}} \tanh \left(\sqrt{\frac{k}{2 l w\left(l^{2}-w^{2}\right)}}\left(l x+\gamma y-\frac{w t^{\alpha}}{\Gamma(1+\alpha)}+\mu\right)\right),
\end{aligned}
$$

where $l, k, \gamma, w, \mu$ are arbitrary constants and $0<\alpha \leq 1$. Figure 2 illustrated the solution $U_{5}$.

Remark 3.1. Applying equation (2.19) to $u_{i}(x, t), i=1,2, \ldots, 8$, we obtain an infinite sequence of solutions of equation (3.1). For illustration, by applying equation (2.19) to $u_{i}(x, t), i$ $=1,2, \ldots, 8$, once, we have new solutions of equation (3.1)

$$
\begin{aligned}
& u_{1,2}^{\star}(x, t)= \\
& \frac{-\frac{k}{2 l w} \pm A_{3} \sqrt{\frac{k}{2 w l}} \tan \left(\sqrt{\frac{k}{2 l w\left(w^{2}-l^{2}\right)}}\left(l x+\gamma y-\frac{w t^{\alpha}}{\Gamma(1+\alpha)}+\mu\right)\right)}{A_{3} \pm \sqrt{\frac{k}{2 w l}} \tan \left(\sqrt{\frac{k}{2 l w\left(w^{2}-l^{2}\right)}}\left(l x+\gamma y-\frac{w t^{\alpha}}{\Gamma(1+\alpha)}+\mu\right)\right)},
\end{aligned}
$$




$$
\begin{aligned}
& u_{3,4}^{\star}(x, t)= \\
& \frac{-\frac{k}{2 l w} \pm A_{3} \sqrt{\frac{k}{2 w l}} \cot \left(\sqrt{\frac{k}{2 l w\left(w^{2}-l^{2}\right)}}\left(l x+\gamma y-\frac{w t^{\alpha}}{\Gamma(1+\alpha)}+\mu\right)\right)}{A_{3} \pm \sqrt{\frac{k}{2 w l}} \cot \left(\sqrt{\frac{k}{2 l w\left(w^{2}-l^{2}\right)}}\left(l x+\gamma y-\frac{w t^{\alpha}}{\Gamma(1+\alpha)}+\mu\right)\right)},
\end{aligned}
$$

$u_{5,6}^{\star}(x, t)=$

$\frac{-\frac{k}{2 l w} \pm A_{3} \sqrt{-\frac{k}{2 w l}} \tanh \left(\sqrt{\frac{k}{2 l w\left(l^{2}-w^{2}\right)}}\left(l x+\gamma y-\frac{w t^{\alpha}}{\Gamma(1+\alpha)}+\mu\right)\right)}{A_{3} \pm \sqrt{\frac{k}{2 w l}} \tanh \left(\sqrt{\frac{k}{2 l w\left(l^{2}-w^{2}\right)}}\left(l x+\gamma y-\frac{w t^{\alpha}}{\Gamma(1+\alpha)}+\mu\right)\right)}$,

$$
\begin{aligned}
& u_{7,8}^{\star}(x, t)= \\
& \frac{-\frac{k}{2 l w} \pm A_{3} \sqrt{-\frac{k}{2 w l}} \operatorname{coth}\left(\sqrt{\frac{k}{2 l w\left(l^{2}-w^{2}\right)}}\left(l x+\gamma y-\frac{w t^{\alpha}}{\Gamma(1+\alpha)}+\mu\right)\right)}{A_{3} \pm \sqrt{\frac{k}{2 w l}} \operatorname{coth}\left(\sqrt{\frac{k}{2 l w\left(l^{2}-w^{2}\right)}}\left(l x+\gamma y-\frac{w t^{\alpha}}{\Gamma(1+\alpha)}+\mu\right)\right)},
\end{aligned}
$$

where $A_{3}, l, k, \gamma, w, \mu$ are arbitrary constants and $0<\alpha \leq$ 1.

\section{The $(3+1)$ dimensional space-time fractional mKDV-ZK equation}

The second equation is the $(3+1)$ dimensional space-time fractional mKDV-ZK equation which has the form ([28])

$$
\begin{aligned}
& D_{t}^{\alpha} u+l u^{2} D_{x}^{\alpha} u+D_{x}^{3 \alpha} u+D_{x}^{\alpha} D_{y}^{2 \alpha} u+D_{x}^{\alpha} D_{z}^{2 \alpha} u=0, \\
& , t>0, \quad 0<\alpha \leq 1,
\end{aligned}
$$

where $l$ is an nonzero constant and $0<\alpha \leq 1$. The mKdV equation is used for representing physical and engineering phenomena such as to describe the ion-acoustic waves in a magnetized plasma, dipole blocking and study of coastal waves in ocean etc., see e.g. [29-31].

Using the transformation

$$
\begin{gathered}
u(x, y, z, t)=U(\xi), \\
\xi=\frac{\beta x^{\alpha}}{\Gamma(1+\alpha)}+\frac{\gamma y^{\alpha}}{\Gamma(1+\alpha)}+\frac{\delta z^{\alpha}}{\Gamma(1+\alpha)}-\frac{w t^{\alpha}}{\Gamma(1+\alpha)},
\end{gathered}
$$

where $\beta, \gamma, \delta$ and $w$ are non zero constants and $0<\alpha \leq 1$. the ODE

Substituting (4.3) with (2.2) and (2.5) into (4.1), we have

$$
\left(\beta^{3}+\beta \gamma^{2}+\beta \delta^{2}\right) U^{\prime \prime \prime}+l \beta U^{2} U^{\prime}-w U^{\prime}=0,
$$

Integrating equation (4.4) once with respect to $\xi$ with the zero constant of integration, we have

$$
\left(\beta^{3}+\beta \gamma^{2}+\beta \delta^{2}\right) U^{\prime \prime}+\frac{l \beta}{3} U^{3}-w U=0
$$

Substituting equations (2.8) into equation (4.5), we obtain

$\left(\beta^{3}+\beta \gamma^{2}+\beta \delta^{2}\right)\left(a b(3-m) U^{2-m}+a^{2}(2-m) U^{3-2 m}+m c^{2} U^{2 m-1}\right.$

$\left.+b c(m+1) U^{m}+\left(2 a c+b^{2}\right) U\right)+\frac{l \beta}{3} U^{3}-w U=0$.

Putting $m=0$, equation (4.6) becomes

$$
\begin{aligned}
& \left(\beta^{3}+\beta \gamma^{2}+\beta \delta^{2}\right)\left(3 a b U^{2}+2 a^{2} U^{3}+b c+\left(2 a c+b^{2}\right) U\right) \\
& +\frac{l \beta}{3} U^{3}-w U=0 .
\end{aligned}
$$

Putting each coefficient of $U^{i}(i=0,1,2,3)$ to zero, we obtain

$$
\left(\beta^{3}+\beta \gamma^{2}+\beta \delta^{2}\right) b c=0,
$$

$$
\left(\beta^{3}+\beta \gamma^{2}+\beta \delta^{2}\right)\left(2 a c+b^{2}\right)-w=0,
$$

$$
\begin{gathered}
3\left(\beta^{3}+\beta \gamma^{2}+\beta \delta^{2}\right) a b=0, \\
2 a^{2}\left(\beta^{3}+\beta \gamma^{2}+\beta \delta^{2}\right)+\frac{l \beta}{3}=0 .
\end{gathered}
$$

Solving equations (4.8)-(4.11), we get

$$
\begin{gathered}
b=0, \\
a c=\frac{w}{2\left(\beta^{3}+\beta \gamma^{2}+\beta \delta^{2}\right)}, \\
a= \pm \sqrt{\frac{-l}{6\left(\beta^{2}+\gamma^{2}+\delta^{2}\right)},}
\end{gathered}
$$

\section{Trigonometric function solutions:}

When $\frac{w}{\beta^{3}+\beta \gamma^{2}+\beta \delta^{2}}<0$, substituting equations (4.12)(4.14) and (4.3) into equations (2.13) and (2.14), we obtain the exact solutions of equation (4.1),

$\tilde{U}_{1,2}(x, y, z, t)=$

$\pm \sqrt{\frac{-3 w}{\beta l}} \tan \left(\sqrt{\frac{w}{2\left(\beta^{3}+\beta \gamma^{2}+\beta \delta^{2}\right)}}\left(\frac{\beta x^{\alpha}}{\Gamma(1+\alpha)}+\frac{\gamma y^{\alpha}}{\Gamma(1+\alpha)}\right.\right.$

$\left.\left.+\frac{\delta z^{\alpha}}{\Gamma(1+\alpha)}-\frac{w t^{\alpha}}{\Gamma(1+\alpha)}+\mu\right)\right)$

and 


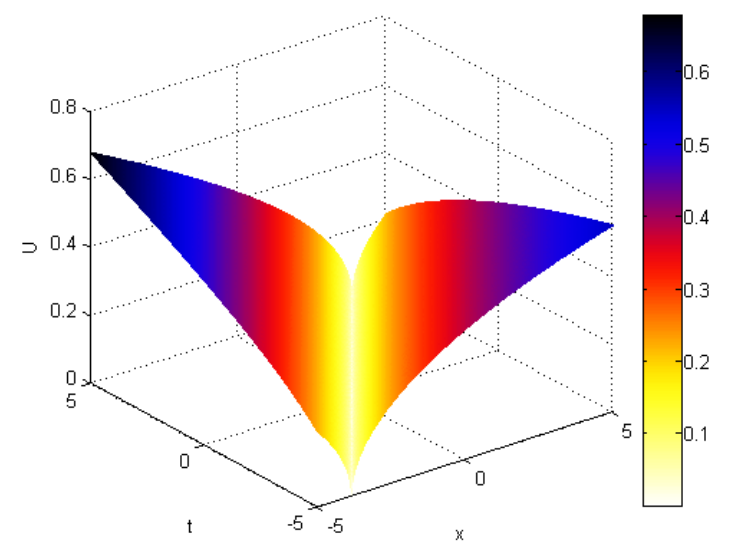

Fig. 3: The solution $\tilde{U}_{1}(x, 0,0, t)$ in (4.15) for $\beta=-2.6, \gamma=-3.5, \delta=3, l=-$ $2, \alpha=1, \mu=0, w=-2$ and $-5 \leq t, x \leq 5$.

$$
\begin{aligned}
& \tilde{U}_{3,4}(x, y, z, t)= \\
& \pm \sqrt{\frac{-3 w}{\beta l}} \cot \left(\sqrt { \frac { w } { 2 ( \beta ^ { 3 } + \beta \gamma ^ { 2 } + \beta \delta ^ { 2 } ) } } \left(\frac{\beta x^{\alpha}}{\Gamma(1+\alpha)}+\frac{\gamma y^{\alpha}}{\Gamma(1+\alpha)}\right.\right. \\
& \left.\left.+\frac{\delta z^{\alpha}}{\Gamma(1+\alpha)}-\frac{w t^{\alpha}}{\Gamma(1+\alpha)}+\mu\right)\right),
\end{aligned}
$$

where $\beta, \gamma, \delta, w$ and $\mu$ are non zero constants and $0<\alpha \leq$ 1. Figure 3 illustrated the solution $\tilde{U}_{1}$.

\section{Hyperbolic function solutions:}

When $\frac{w}{\beta^{3}+\beta \gamma^{2}+\beta \delta^{2}}>0$, substituting equations (3.12)(3.14) and (4.3) into equations (2.15) and (2.16), we obtain exact solutions of equation (4.1),

$$
\begin{aligned}
& \tilde{U}_{5,6}(x, y, z, t)= \\
& \pm \sqrt{\frac{3 w}{\beta l}} \tanh \left(\sqrt { \frac { - w } { 2 ( \beta ^ { 3 } + \beta \gamma ^ { 2 } + \beta \delta ^ { 2 } ) } } \left(\frac{\beta x^{\alpha}}{\Gamma(1+\alpha)}\right.\right. \\
& \left.\left.+\frac{\gamma y^{\alpha}}{\Gamma(1+\alpha)}+\frac{\delta z^{\alpha}}{\Gamma(1+\alpha)}-\frac{w t^{\alpha}}{\Gamma(1+\alpha)}+\mu\right)\right)
\end{aligned}
$$

and

$$
\begin{aligned}
& \tilde{U}_{7,8}(x, y, z, t)= \\
& \pm \sqrt{\frac{3 w}{\beta l}} \operatorname{coth}\left(\sqrt { \frac { - w } { 2 ( \beta ^ { 3 } + \beta \gamma ^ { 2 } + \beta \delta ^ { 2 } ) } } \left(\frac{\beta x^{\alpha}}{\Gamma(1+\alpha)}+\frac{\gamma y^{\alpha}}{\Gamma(1+\alpha)}\right.\right. \\
& \left.\left.+\frac{\delta z^{\alpha}}{\Gamma(1+\alpha)}-\frac{w t^{\alpha}}{\Gamma(1+\alpha)}+\mu\right)\right),
\end{aligned}
$$

where $\beta, \gamma, \delta, w$ and $\mu$ are non zero constants and $0<\alpha \leq$ 1. Figure 4 illustrated the solution $\tilde{U}_{5}$.

Remark 4.1. Similarly as shown in Remark (3.1), we can give an infinite solutions of equation (4.1).

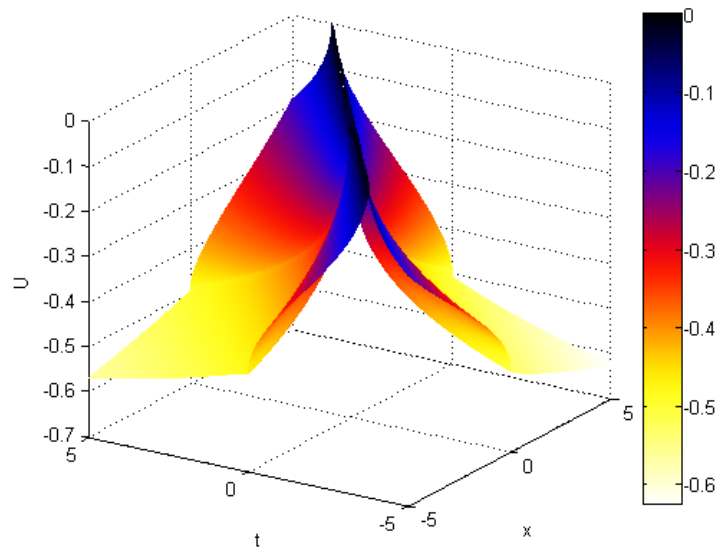

Fig. 4: The solution $\tilde{U}_{5}(x, 0,0, t)$ in (4.17) for $\beta=2.6, \gamma=-3.5, \delta=3, l=$ $2, \alpha=0.5, \mu=0, w=3$ and $-5 \leq t, x \leq 5$.

\section{Remark 4.2.}

1. Comparing our results concerning equation (3.1) with the results in [27, 32], one can see that our results are new and most extensive.

2. Comparing our results concerning equation (4.1) with the results in $[31,33]$, one can see that our results are new and most extensive.

3. Comparing our solutions for equations (3.1) and (4.1) with [27, 31-33], it can be seen that by choosing suitable values for the parameters similar solutions can be verified.

4. Actually the Riccati-Bernoulli Sub-ODE technique has a very important feature, that admits infinite sequence of solutions of equation, which is explained clearly in Section 2.1. In fact this feature has never given for any another method.

5. Consequently, the method is efficacious, robust and adequate for solving other type of space-time fractional differential equations.

\section{Conclusions}

In this work, a Riccati-Bernoulli Sub-ODE technique has successfully been applied to exact solutions for the nonlinear fractional Zoomeron equation and the $(3+1)$ dimensional space-time fractional mKDV-ZK equation with modified Riemann-Liouville derivative. Fractional complex transform is also used as the basic ingredient to obtain exact solutions for these nonlinear equations. As a result, some new exact solutions for them have successfully 
been obtained. The graphs of some solutions are depicted for suitable coefficients. Actually this method can be applied for many other nonlinear FDEs appearing in mathematical physics and natural sciences.

Acknowledgement: The author wants to thank the editor and reviewers for valuable comments.

\section{References}

[1] K.S. Miller and B. Ross, An Introduction to the Fractional Calculus and Fractional Differential Equations. Wiley: New York, 1993.

[2] I.Podlubny, Fractional Differential Equations. Academic Press: California, (1999).

[3] A.A. Kilbas, H.M. Srivastava and J.J. Trujillo, Theory and Applications of Fractional Differential Equations. Elsevier: Amsterdam, (2006).

[4] I. Podlubny, Fractional Differential Equations. Academic Press: San Diego, (1999).

[5] S. Zhang and H-Q. Zhang, Fractional sub-equation method and its applications to nonlinear fractional PDEs. Physics Letters A, 375(2011), 1069-1073.

[6] B. Tong, Y. He, L. Wei and X. Zhang, A generalized fractional sub-equation method for fractional differential equations with variable coefficients. Physics Letters A, 376(2012), 2588-2590.

[7] S. Saha Ray and S. Sahoo, A novel analytical method with fractional complex transform for new exact solutions of timefractional fifth-order Sawada-Kotera equation, Reports on Math. Phys., 75(1) (2015), 63-72.

[8] N. Shang and B. Zheng, Exact Solutions for Three Fractional Partial Differential Equations by the $\left(\frac{G}{G}\right)$ Method, Int. J. of Appl. Math., 43(3) 2013, 1-6.

[9] B. Zheng, $\left(\frac{G}{G}\right)$-expansion method for solving fractional partial differential equations in the theory of mathematical physics. Communications in Theoretical Physics, 58 (2012), 623-630.

[10] B. Lu, The first integral method for some time fractional differential equations. Journal of Mathematical Analysis and Applications 395 (2012), 684-693.

[11] S.M. Ege and E. Misirli, The modified Kudryashov method for solving some fractional-order nonlinear equations, Advances in Difference Equations, 2014, 2014, 135.

[12] S. Zhang, Q-A. Zong, D. Liu and Q. Gao, A generalized expfunction method for fractional riccati differential equations. Communications in Fractional Calculus 1(1) (2010), 48-51.

[13] A. Bekir, o. Guner and A.C. Cevikel, The fractional complex transform and exp-function methods for fractional differential equations, Abstr. and Appl. Anal., 2013, 2013 426-462.

[14] Z.Z. Ganjia, D.D. Ganjia and Y. Rostamiyan, Solitary wave solutions for a time-fraction generalized Hirota-Satsuma coupled KdV equation by an analytical technique, Applied Mathematical Modelling 33 (2009), 3107-3113.

[15] W. Liu and K. Chen, The functional variable method for finding exact solutions of some nonlinear time-fractional differential equations, Pramana J. Phys., 81(2013), 377-384.
[16] M.A.E. Abdelrahman and M.A. Sohaly, Solitary Waves for the Modified Korteweg-De Vries Equation in Deterministic Case and Random Case. J Phys Math. 8(1) (2017), [DOI: 10.4172/2090-0902.1000214].

[17] M.A.E. Abdelrahman and M.A. Sohaly, Solitary waves for the nonlinear Schrödinger problem with theprobability distribution function in the stochastic input case, Eur. Phys. J. Plus, (2017) 132: 339.

[18] X.F. Yang, Z.C. Deng and Y. Wei, A Riccati-Bernoulli sub-ODE method for nonlinear partial differential equations and its application, Adv. Diff. Equa. 1 (2015) 117-133.

[19] B. Zheng, A new Bernoulli sub-ODE method for constructing traveling wave solutions for two nonlinear equations with any order, U. P. B. Sci. Bull., Series A, 73(3), (2011).

[20] M. Inc, A. I. Aliyu and A. Yusuf, Traveling wave solutions and conservation laws of some fifth-order nonlinear equations. Eur. Phys. J. Plus, 132 (2017) 224.

[21] F. Xu and Q. Feng, A generalized sub-ODE method and applications for nonlinear evolution equations. J. Sci. Res. Report 2 (2013), 571-581.

[22] M. Caputo, Linear models of dissipation whose $Q$ is almost frequency independent II, Geophys. J. Royal Astronom. Soc. 13 (1967), 529-539.

[23] S. G. Samko, A. A. Kilbas and O. I. Marichev, Fractional Integrals and Derivatives: Theory and Applications, Gordon and Breach, Yverdon, (1993).

[24] G. Jumarie, Modified Riemann-Liouville derivative and fractional Taylor series of nondifferentiable functions further results, Comput. Math. Appl. 51 (2006), 1367-1376.

[25] G. Jumarie, Table of some basic fractional calculus formulae derived from a modified Riemann-Liouvillie derivative for nondifferentiable functions. Applied Mathematics Letters 22 (2009), 378-385.

[26] J. H. He, S. K. Elegan and Z. B. Li, Geometrical explanation of the fractional complex transform and derivative chain rule for fractional calculus, Phys. Lett. A 376 (2012), 257-259.

[27] O. Guner, A. Bekir and H. Bilgil, A note on exp-function method combined with complex transform method applied to fractional differential equations, Adv. Nonlinear Anal., 4(3) (2015), 201-208.

[28] R.L. Mace and M.A. Hellberg, The Korteweg-de Vries-ZakharovKuznetsov equation for electron-acoustic waves, Phys. Plasmas 8(6) (2001), 2649-2656.

[29] F. Demontis, Exact solutions of the modified Korteweg-de Vries, Theoret. Math. Phys., 168 (1) (2011), 886-897.

[30] I. Aslan, Exact solutions of a fractional-type differential-difference equation related to discrete MKdV equation, Commun. Theor. Phys. 61 (2014), 595-599.

[31] S. Sahoo and S.S. Ray, Improved fractional sub-equation method for $(3+1)$-dimensional generalized fractional KdVZakharov-Kuznetsov equations, Comput. Math. Appl. 70 (2015), 158-166.

[32] Marwan Alquran and Kamel Al-khaled, Mathematical methods for a reliable treatment of the $(2+1)$-dimensional Zoomeron equation, Math. Sci. 6 (12) (2012).

[33] O. Guner, E. Aksoy, A. Bekir and A.C. Cevikel, Different methods for (3+1)-dimensional space-time fractional modified KdVZakharov-Kuznetsov equation. Comput Math Appl. 71 (2016), 1259-1269. 